\title{
Genetic family history questionnaire ${ }^{1}$
}

JUNE COLE, P. MICHAEL CONNEALLY, M. E. HODES, AND A. D. MERRITT

From the Department of Medical Genetics, Indiana University School of Medicine, Indianapolis, Indiana 46202, USA

SUMMARY A written questionnaire suitable for obtaining comprehensive genetic family data was developed. The questionnaire was designed to solicit information on the name, date of birth, sex, health problems, date of death (if deceased), spouse, abortions, stillbirths, and offspring of the patient plus first, second, and some third degree relatives of the patient.

The questionnaire was evaluated for its effectiveness in obtaining an accurate and complete family history. During the first phase, 77 completed questionnaires were continuously assessed, and the questionnaire was modified until the present version evolved. Using the present version, 60 completed questionnaires disclosed only 15 problems, 10 of them minor.

The questionnaire was also designed in conjunction with a computer programme developed for the entry of pedigree data. This programme is part of the computer system, MEGADATS, which is used for the acquisition, storage, and manipulation of genetic family data.

The questionnaire offers these advantages over the pedigree obtained at the time of the clinic visit: $\vec{\varphi}$ (1) permits consultation with other family members, (2) saves clinic time, (3) gives prior knowledge of $\infty$ reason for clinic appointment, (4) anamnestic infallibility, (5) time is available to check for family o record linkage, (6) computer adaptability for entering pedigree data, and (7) basic format can be modified easily. A modified sample questionnaire is presented in the Appendix.

As the demand for genetic counselling services increases, more efficient methods to ensure the accurate and complete acquisition of genetic family data in the easiest possible way must be developed. Also, as computer facilities are being used more frequently to help expedite the processing of data, new methods must take into account the restrictions placed upon data collection so they will be in a format suitable for ease of entry into the computer system. The development and use of a written family history questionnaire which was designed in conjunction with an interactive computer programme developed to enter family data, code the data, and draw a family pedigree represents one method of facilitating the collection of family data.

In this paper we discuss the design of the questionnaire, its use by the patients and clinical personnel in the Department of Medical Genetics at Indiana University School of Medicine, and its relation with the computer system, MEGADATS, developed

'This is publication No. 77-21 from the Department of Medical Genetics and was supported in part by the Indiana University Human Genetics Center PHS GM 2 1054, Dental Genetics Training Grant PHS DE 00007, and Human Genetics Data Resources PHS MB 00109.

Received for publication 19 April 1977 for the acquisition, storage, and manipulation of genetic family data (Merritt et al., 1976).

\section{Subjects and methods}

A questionnaire was sent to all clinic families scheduled for an initial appointment at the Medical Genetics Clinic between August 1975 and December 1976 when there was at least a 2-week interval between the $₹$ date of scheduling and the appointment. Respondants $ᄋ$ were requested to complete the form and return it before their clinic appointment. The questionnaire was designed to include the individuals shown in Fig. 1. The dark lines indicate individuals ascertained on 0 every basic pedigree-patient, spouse, children, sibs $N$ and half-sibs, nieces, nephews, parents, aunts, uncles, N first cousins, and grandparents - while the light lines indicate individuals ascertained only if they were affected, for example, the patient's great uncle. Also $\stackrel{\circ}{=}$ included is a question about the patient's reason for making an appointment.

A portion of the questionnaire is shown in Fig. 2. An abbreviated version of the questionnaire is shown $\overrightarrow{\mathbb{D}}$ in the Appendix. This particular section is for $\frac{}{\mathbb{D}}$ 


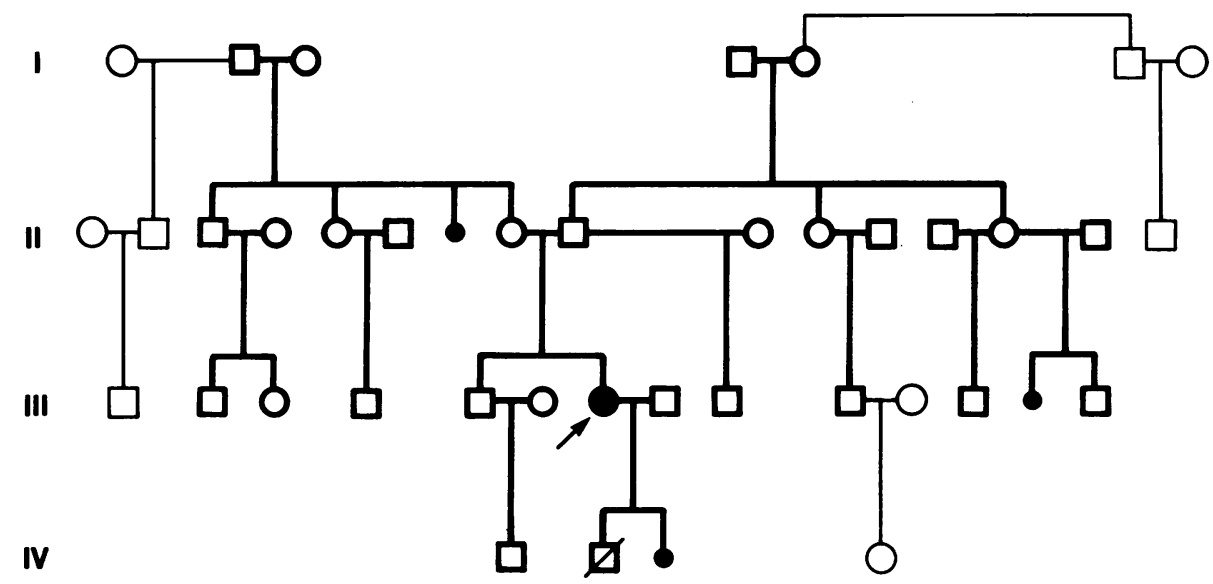

Fig. 1 Sample pedigree ascertained by the use of the questionnaire. The dark lines indicate individuals ascertained on every basic pedigree. The light lines indicate individuals ascertained only if they were affected.

information about the maternal grandparents, aunts, uncles, and cousins. The query concerning the maternal grandmother (the second part of question number one) illustrates the questions asked about each person in the pedigree: last name, maiden name, first name, date of birth, status (if deceased, the cause and age at death), and health problems. The place of birth is obtained only for the grandparents to give a general idea of the background of the family. Information concerning spontaneous and induced abortions and stillbirths is also obtained.

In question no. 2 , information is ascertained about the oldest maternal aunt or uncle and the name and date of birth of the spouse. The health problems of these people are asked immediately after their names. In question no. 3 , information is ascertained about the cousins of the patient. In addition to the essential information listed above, the name and date of birth of the other parent are obtained. In this way multiple mates are ascertained but only if they had children.

A family history containing the health problems of each individual in the family was constructed from the questionnaire and was included in the patient's record. This record was available for review by the clinic staff before the patient's appointment. Patients (or their parents) as well as the clinic personnel were interviewed about the use of the questionnaire, and it was continually modified based on these responses until the present version evolved. The latter was evaluated as to its effectiveness in ascertaining a complete and accurate pedigree by comparing the pedigree constructed from the questionnaire with the final pedigree. The final pedigree is the pedigree drawn from the questionnaire which has been verified for accuracy and completeness and revised if necessary during the clinic visit.

\section{Results}

A total of 190 questionnaires were posted to clinic patients between August 1975 and December 1976. These were divided into 2 groups. The first group of 104 questionnaires was used to determine the revisions that should be made to make the form more effective. The second group of 86 questionnaires was used to evaluate the accuracy and completeness of the information ascertained from the present version of the questionnaire.

Of the 104 questionnaires in the first group, 80 were returned. Of these 80 patients, 3 failed their clinic appointments and 12 of the 104 patients came to the clinic without completing the form. A pedigree, constructed from the information ascertained by the questionnaire, was used in the preclinic evaluation of the patient and placed in the family record.

All the pedigrees were checked for accuracy and completeness, and the families were interviewed to ascertain their reactions to the use of the questionnaire. Questions were asked about the following: (1) Problems or questions they had while they were completing the form, (2) omission from the family history of anyone who should have been included, (3) consultation with other family members for additional information, and (4) the amount of time required to complete the form. Answers to the first two questions changed as the form was modified. However, the answers to the last two questions also apply to the present version of the questionnaire. A majority of these patients $(82 \%)$ consulted with other family members for accurate information about dates of birth, maiden names, and given names. One-third of the patients $(32 \%)$ required more than 2 hours, $60 \%$ required 1 to 2 hours, and only $8 \%$ required less than 1 hour to complete the form. 
SECTION 7: Information about the Patient's Mother's Parents, Brothers and Sisters.

1. Name of Patient's Mother's Father, Last/First: Birthdate, Mo/Day/Yr: Living, Yes/No:

If deceased, age at death: Place of Birth, City/State.

Name of Patient's Mother's Mother, Last (Maiden) First: Birthdate, Mo/Day/Yr: Living, Yes/No: If deceased, age at death: Place of Birth, City/State.

Please list any health problems and, if deceased, give the cause of death.

Father

Mother

Did these parents have any miscarriages, stillbirths, or therapeutic abortions? Yes No _ _ If yes, give the number of each, the year each occurred, the month of pregnancy during which any miscarriage occurred, and the reason for any therapeutic abortion.

2. List each of the children with these same two parents starting with the oldest child (do not include the patient's mother). Do not include children with the above listed mother and a different father or the above listed father and a different mother.

First Child: Name: Birthdate, Mo/Day/Yr: Sex, M/F: Living, Yes/No: If deceased, age at death: Married, Yes/No: If married, name (use maiden name if female) and birthdate of spouse.

Please describe any health problems either of these people may have and, if deceased, give the cause of death.

3. List all the children of this person. Name of Child, Last/First: Birthdate, Month/Day/Yr: Sex: M/F: Living, Yes/No: If deceased, age at death: Name and birthdate of other parent (use maiden name if other parent is the mother).

1.

2.

3.

4.

5.

Fig. 2 Page 6 of the questionnaire. For explanation, see text. 
Of the 86 questionnaires in the second group, 61 were completed. One of these 61 patients failed to keep his appointment and an additional 4 of the 86 patients came to the clinic without completing the form. The accuracy and completeness of the pedigree constructed from the questionnaire was compared with the final pedigree in the 60 cases where the patients returned the forms and also kept their appointments.

The accuracy and completeness of the pedigrees drawn from the questionnaire were compared with the final pedigrees and may be summarised as follows: (1) the basic pedigree, shown by the dark lines in Fig. 1, required no changes and additional information about more distant relatives, shown by the light lines in Fig. 1 , could be drawn from the information given in the questionnaire (8 pedigrees). (2) the basic pedigree required no changes, but additional information about more distant relatives could not be added until their exact relationship was determined at the time of the clinic appointment (11 pedigrees). (3) The basic pedigree required no changes, and there was no information about more distant relatives (21 pedigrees). (4) The basic pedigree required no changes, but only the husband's or wife's side of the family was ascertained. The other side of the family was added during the clinic appointment (5 pedigrees). (5) Minor changes were required (10 pedigrees). (6) Major changes were required ( 5 pedigrees).

Minor changes included the addition of approximate ages and dates of birth, the addition, in 2 cases, of uncles who had died at an early age from unknown causes, the addition of an uncle and his family because there was not enough space on the form and the information was not added on the back of the form, one case in which clinodactyly and dental problems and one case in which polydactyly was not included because the patients did not think that they were health problems.

Major problems occurred in only 5 cases and included leaving part of the questionnaire blank in 3 cases and writing the information in their own way on the back of the form rather than following the format of the questionnaire in 2 cases. In 2 of the 3 cases in which one side of the family was omitted, an affected person was also omitted. These were the only affected individuals added at the clinic appointment except for the people with clinodactyly and dental problems and those with polydactyly mentioned above.

\section{Discussion}

At the present time pedigree data and diagnostic information is collected on approximately 50000 people per year by the Department of Medical Genetics. The average pedigree contains approxi- mately 30 individuals, and information on over 350000 people is stored in the Medical Genetics departmental data base.

Before designing the questionnaire, a survey was made of existing questionnaires to determine whether one was available which would satisfy the requirements for the extensive family data currently being collected. Though questionnaires have been developed for specific uses, a search of the literature revealed no questionnaires suitable for obtaining comprehensive family histories.

The sample page shown in Fig. 2 illustrates the general approach used in designing the questionnaire. After the information about the maternal grandparents is supplied, all the children of these two people are ascertained. We have found it essential to indicate that this means all the children of this mating only and not children with either a different mother or a different father. If this is not clearly indicated it is impossible to know if the children share the same parents. We have also found that it is important to ask repeatedly for health problems to eliminate missing affected individuals.

The question about the name and date of birth of the other parent in question 3 allows multiple mates of the aunt or uncle to be ascertained if children resulted from that union. It requires much less space to ask this type of question than to ask for all mates of all aunts and uncles and then all the children of each of these matings.

General questions are asked about the health problems of more distant members of the family. These people are shown in light lines in Fig. 1. If one of these people is affected, his relation to the patient is ascertained. In this way, affected people are not missed. Because of the use of married names and incorrect relations (for example second cousin of the patient indicated when first cousin once removed is really meant), these people often cannot be drawn on the pedigree without further information. A longer questionnaire could be designed to include more complete information on these people, but the form would be 3 times as long and little more information would be obtained.

In using the questionnaire we have found that the name of the 'patient' should be written on the form since the mother often thinks she is the patient.

The definition of 'accurate and complete' was dependent upon the thoroughness of the interviewer. In some cases, a pedigree accepted as accurate and complete by one interviewer might be extended to include many other people by another interviewer. If there was no clinical benefit in extending the form the pedigree as drawn from the questionnaire was considered accurate and complete. If, however, major or minor changes were made as described in the 
Results section, the pedigree was not considered accurate and complete.

In our experience, the questionnaire has a number of advantages over the pedigree taken at the time of the clinic visit. These are: (1) Patients complete the forms at their own pace and consult with other members of the family, if necessary, to obtain accurate information. (2) Unique family information is available before the clinic appointment, thus expediting investigation of the genetic disorder. (3) The patients' reasons for coming to the clinic are known and give an indication of their understanding of the problems, their expectations, and possible misconceptions. (4) the questionnaire does not 'forget' to ask a question. (5) Time is available to check the existing data base for family record linkage. (6) Clinic time required for obtaining a basic pedigree is reduced. The disadvantages of the questionnaire are its length (15 pages) and the time required to complete it ( 1 to 2 hours). However, we have been delighted and surprised to find that most families complete the form without objecting to either the length of the form or the time required to complete it.

The questionnaire was also designed in conjunction with an interactive computer programme developed to enter and code family data and draw a family pedigree. The information on the questionnaire is entered page by page as the individual screens appear on the computer terminal. Each person has one screen and all essential information plus comments are entered. This pedigree input programme is part of a large computer system called Medical Genetics
Acquisition and Data Transmission System $\mathbb{\Phi}$ (MEGADATS). In addition to the interactive pedigreed programme, MEGADATS also includes a name: search programme to determine if a person in a new family can be linked through family record linkage too another family already in our files. It also includeso specific programmes for the input and retrieval of diagnostic and laboratory data.

The basic format of the questionnaire has been modified for specific uses. For example, a question- ${ }^{\infty}$ naire has been developed for Huntington disease $\vec{O}$ which would be useful for any autosomal dominant $\overrightarrow{-}$ disorder in which information is desired on one side of the family through as many as five generations as well as information such as age of onset and firsto symptoms of the disease. Another version has been $\vec{G}$ developed for prenatal diagnosis. The latter is a short version of the basic pedigree but has additional questions relating to the mother's pregnancy. These $\mathrm{O}$ will be described elsewhere.

\section{Reference}

Merritt, A. D., Kang, K. W., Conneally, P. M., Gersting, J. M., and Rigo, T. (1976). MEGADATS: A computer system for family data acquisition, storage, and analysis. In Registers for the $\overrightarrow{0}$ Detection and Prevention of Genetic Disease, pp. 31-51. Symposia Specialists, Miami.

Requests for reprints to Dr June Cole, Department of Medical Genetics, Indiana University School of $\frac{\bar{O}}{\mathrm{O}}$ Medicine, 1100 West Michigan Street, Indianapolis, ֶ Indiana 46202, USA.

Appendix

The following is a copy of the letter and an abbreviated version of the family history questionnaire which are sent to clinic patients. The information written in italics indicates that questions and spaces for the answers to them should be added to expand the form to its original length of 15 pages.
Copies of the questionnaire may be obtained from Dr June Cole, Department of Medical Genetics, Indiana. University School of Medicine, 1100 West Michigan Street, Riley Research 129, Indianapolis, $\frac{9}{3}$ Indiana 46202.

\section{Dear}

An accurate family history is needed to help us understand the inheritance of genetic disorders. In order to make maximum use of your time while you are at the Medical Genetics Clinic, we ask that you complete the enclosed family history form and return it in the enclosed envelope so that it will reach us a week before your clinic appointment. The purpose of having you complete the family history information before you come to the clinic is to enable you to complete the form at your own leisure and to allow us to have some preliminary information about your family history prior to your appointment. This information is confidential.

Please answer all of the questions as completely and accurately as possible. If you do not know all of the information asked about a specific person, give any information you do know. If you need additional space to answer any question or if you wish to add any supplemental information, please write any such information on the back of the page, indicating the person's name. It is especially important to use maiden names whenever possible and to indicate any health problems that any members of the family may have or, if deceased, the cause of death. 
If you have any questions, please call the appointments clerk. Department of Medical Genetics. at the telephone number printed above.

Thank you for your cooperation.

Sincerely

Family No.

FAMILY HISTORY QUESTIONNAIRE

(Department of Medical Genetics, Indiana University School of Medicine)

Hospital No.

SECTION 1: Information about the patient.

Name of Patient, Last (Maiden), First: Birthdate, Mo/Day/Yr: Sex, M/F: Living, Yes/No: If deceased, age at death: Married, Yes/No: Name of Spouse: Birthdate of Spouse.

Is the patient (or spouse) pregnant at this time? Yes No If yes, when is the estimated date of delivery?

Name of person completing this family history and relationship to the patient.

Name

(city, state, zip code)

Date

Relationship to the patient.

Name and address of referring physician:

Have any of the patient's relatives ever participated in any study or been to the Medical Genetics Clinic at Indiana University Medical Center? Yes No If yes, give the name of the person, his or her birthdate and the person's parents' names.

Please list any health problems the patient has and, if deceased, give the cause of death.

Please list any hospitalization (dates, places and reasons).

Hospital name and location

Reason

Date of hospitalization

Information about the patient's children:

Name of Child, Last, First: Birthdate, Mo/Day/Yr: Sex, M/F: Living, Yes/No: If deceased, age at death.

Name of other parent (patient's spouse): Birthdate of other parent. 
Please describe any health problems these children may have and, if deceased, give the cause of death.

This question will be referred to as 'health problems' on subsequent pages.

Did the patient or his spouse have any miscarriages, stillbirths, or therapeutic abortions? Yes No

If yes, give the number of each, the year each occurred, the month of the pregnancy during which any miscarriage occurred, and the reason for any therapeutic abortion.

This question will be referred to as 'miscarriages' on subsequent pages.

SECTION 2: Information about the parents of the patient. Name (Father), Last, First: Birthdate, Mo/Day/Yr: Living, Yes/No: If deceased, age at death: Place of Birth, City/State: Race and Religion.

Name (Mother), Last (Maiden), First: Birthdate, Mo/Day/Yr: Living, Yes/No: If deceased, age at death: Place of Birth, City/State: Race and Religion.

SECTION 3: Information about the brothers and sisters of the patient. List all of the other children in the family with the same two parents as the patient starting with the oldest child (do not include the patient). Half-brothers and half-sisters of the patient will be listed in another section. Name of child, First: Birthdate, Mo/Day/Yr: Sex, M/F: Living, Yes/No: If decreased, age at death: Married, Yes/No: If married, Name of Spouse (use maiden name if female): Birthdate of Spouse:

1.

Spaces are left for 5 children.

\section{'Health Problems'}

\section{'Miscarriages'}

SECTION 4: Information about the nieces and nephews of the patient. If any of the children listed in SECTION $\overrightarrow{\vec{O}}$ 3 or their spouses have had a child, miscarriage, stillbirth, or therapeutic abortion, please answer the following 3 for each child starting with the oldest child.

Name of person listed in SECTION 3. This question is repeated three times.

List all of the children of this person starting with the oldest child. Name of Child, Last, First: Birthdate, Mo/Day/Yr: Living, Yes/No: If deceased, age at death: Name and birthdate of other parent (use maiden name $\bar{O}$ if the other parent is the mother of the child).

1. Spaces are left for 5 children.

'Health Problems'

'Miscarriages'

SECTION 5: Half-brothers and half-sisters of the patient (Father's side of the family). Does the patient's father have any other children? Yes _ No _ . If yes, please list these children starting with the oldest. Name of Child, Last, First: Birthdate, Mo/Day/Yr: Sex, M/F: Living, Yes/No: If deceased, age at death: Name of Mother, Last (Maiden) First: Birthdate of Mother, Mo/Day/Yr.

1. Spaces are left for 5 children. 


\section{'Health Problems'}

'Miscarriages'

Have any of the children listed in SECTION 5 had children of their own? Yes No If yes what is the total number of children? If yes, do (or did) any of these children have health problems? Yes _ No __ If yes, give name of the child, his/her birthdate, sex; parents' names (maiden name of the mother) and briefly describe the health problems. If the child is deceased, give the cause of death.

SECTION 6: Half-brothers and half-sisters of the patient (Mother's side of the family).

Does the patient's mother have any other children? Yes No If yes, please list these children starting with the oldest.

Name of Child, Last, First: Birthdate, Mo/Day/Yr: Sex, M/F: Living, Yes/No: If deceased, age at death: Name of Father, Last, First: Birthdate of Father, Mo/Day/Yr.

1.

Spaces are left for 5 children.

\section{'Health Problems'}

\section{'Miscarriages'}

Have any of the children listed in SECTION 6 had children of their own? Yes No If yes, what is the total number of children? __. If yes, do (or did) any of these children have health problems? Yes No _ I If yes, give the name of the child, his/her birthdate, sex, parents' names (use maiden name for mother) and briefly describe the health problems. If the child is deceased, give the cause of death.

SECTION 7: Information about the Patient's Mother's Parents, Brothers, Sisters. Name of Patient's Mother's Father, Last/First: Birthdate, Mo/Day/Yr: Living, Yes/No: If deceased, age at death: Place of Birth, City/State.

This section is repeated for the patient's Father's Parents, Brothers \& Sisters.

Name of Patient's Mother's Mother, Last (Maiden) First: Birthdate, Mo/Day/Yr: Living, Yes/No: If deceased, age at death: Place of Birth, City/State.

\section{'Health Problems'}

\section{'Miscarriages'}

List each of the children with these same parents starting with the oldest child (do not include the patient's mother). Do not include children with the above listed mother and a different father or the above listed father and a different mother.

First Child: Name: Birthdate, Mo/Day/Yr: Sex, M/F: Living, Yes/No: If deceased, age at death: Married, Yes/No: If married, Name (use maiden name if female) and birthdate of spouse.

This question is repeated for 5 children.

\section{'Health Problems'}

List all of the children of this person. Name of Child, Last/First: Birthdate, Mo/Day/Yr: Sex, M/F: Living, Yes/No: If deceased, age at death: Name and birthdate of other parent (use maiden name if other parent is the mother):

1. 


\section{'Health Problems'}

\section{'Miscarriages'}

Have any of the children listed in SECTION 7, (above question) had children of their own? Yes No

If yes, what is the total number of children? If yes, do (or did) any of these children have health problems? Yes _ No __ If yes, give the name of the child, his/her birthdate, sex, parents' names (use maiden name for mother) and briefly describe the health problems. If the child is deceased, give the cause of death.

Does the patient's father's father have any other children? Yes their children or grandchildren have any health problems? Yes No No If yes, do any of these children, $\vec{\circ}$ the health problems.

This is repeated for the maternal side of the family.

Does the patient's father have any grandparents, uncles aunts or cousins that have or did have any health problems- Yes No If yes, give the name of the person, their birthdate, sex, parents' names (use maiden name if female), relationship to the patient (be as detailed as necessary to show the precise relationship), and briefly describe the health problems.

\section{This question is repeated for the remaining grandparents.}

Is there anyone else in your family that you feel should have been included in the family history? Yes No __ If yes, please give their name, their parents' names (use maiden name if female), sex, relationship to $\overrightarrow{0}$ the patient (be as detailed as necessary to show the precise relationship), and briefly describe their health problems.

Were any of the parents listed in the family history related before marriage? (For example, were they second cousins?) Yes No If yes, give their names and their relationship before marriage.

Please describe your reasons for your appointment with the Department of Medical Genetics. For example, what type of information do you hope to receive and what questions do you want answered? 